\title{
Health care needs need to be focused on health
}

\author{
Johannes Bircher ${ }^{1}$, Karl-Heinz Wehkamp ${ }^{2}$ \\ ${ }^{1}$ Hepatology, Department of Clinical Research, University of Bern, Bern, Switzerland; jbi@swissonline.ch \\ ${ }^{2}$ Life Sciences Faculty, HAW Hamburg, Campus Bergedorf, Lohbrügger Kirchstr, Hamburg, Germany; karl.wehkamp@t-online.de
}

Received 15 April 2011; revised 10 May 2011; accepted 23 May 2011.

\begin{abstract}
In the past decades health care and medicine in most countries got more or less in a state of crisis. This is not surprising because, so far, there is no consensus about the nature of health. This shortcoming inhibits constructive, interdisciplinary dialogues about health values. It renders priority setting controversial and subject to power struggles. A new definition of health, known as the Meikirch Model, could correct this deficiency. It states: "Health is a dynamic state of wellbeing characterized by a physical, mental and social potential, which satisfies the demands of a life commensurate with age, culture, and personal responsibility. If the potential is insufficient to satisfy these demands the state is disease." The potential is composed of a biologically given and a personally acquired component. Thus this definition characterizes health with six essential features, which are suitable for an analysis of and priority setting in medical consultations and in health care policy decisions. A wide discussion about this definition of health followed by its implementation is expected to render health care individually and socially more beneficial.
\end{abstract}

Keywords: Health; Definition of Health; Health Care Systems; Health Care Needs; Meikirch Model

\section{INTRODUCTION}

In most countries health care systems are now in a chronic state of crisis. This is due to many reasons, yet a rapid increase in costs and a change in values appear to be predominant. Physicians have lost control of health care in favor of economists and politicians. As a result leadership is no longer based on medical but on economic and political values. In health personnel this has created much insecurity and frustration.

Currently, the predominant question is how to redirect the self-organization of health care systems and how to integrate them into the respective societies. Management of complexity is a challenge that is well known in large organizations. Experienced leaders generally focus with great care on the "product" their organization has to realize. They insist that all persons within their realm of influence know exactly what the purpose of their organization is. Yet, when it comes to health care, it is astonishing that the very purpose of the system, i.e. "health" cannot be described. Everybody speaks with respect about health, yet no one can explain what it really is. As a result, in most health care systems, the level of misunderstanding and conflict is high. This can be exemplified by the following statement: "Patients are customers and physicians service providers.” This obviously is the opinion of economists, yet most physicians and patients would wholeheartedly disagree. Many such misunderstandings may be found at all levels of health care systems. In order to guard against them, a new understanding about the terms of health and disease must be created.

In 1946 WHO declared its famous definition of health [1]. It reads as follows: "Health is a state of complete physical, mental and social well-being and not merely the absence of disease or infirmity.” At that time this was a significant advance because for the first time it officially postulated the importance of mental and social factors for health. Since then, however, our understanding of physical conditions, mental functioning and social wellbeing has markedly evolved. As a result the WHO definition is now considered to be idealistic to an extent that almost no one can consider him or herself to be healthy. Consequently, agreement with this definition has become limited and, at the present time, it can no longer serve as a central concept for the implementation of health care systems. We now need a concept of health that respects the dignity of each person, distinguishes between health and disease, provides essential elements for the process of diagnosis and reimbursement and clarifies the relationship between individual, and society.

An analysis of different descriptions of health reveals 
that most of them have merits and validity from certain points of view and limitations from others [2]. This may be explained by the fact that it is difficult to develop concepts with sufficient interdisciplinary merits. Most authors know their own fields best and attach less importance to others. The definition, which has become known as the Meikirch Model, is based on experiences reflected by physicians [3]. It uses six specific criteria to describe health, all of which may be surveyed or investigated when examining human beings or health care. Consequently, the Meikirch Model appears to be suitable for application in medicine and in health care systems.

\section{DESCRIPTION OF THE MEIKIRCH MODEL [4,5]}

Health is defined as follows: "Health is a dynamic state of wellbeing characterized by a physical, mental and social potential, which satisfies the demands of a life commensurate with age, culture, and personal responsibility. If the potential is insufficient to satisfy these demands the state is disease.” The latter includes sickness, illness, ill health, and malady.

In this definition the potential consists of two components, a biologically given and a personally acquired potential (Figure 1). At birth the biologically given potential has a finite value. Thereafter it decreases throughout life and at the time of death it is zero. In contrast, the personally acquired potential is quite small at the time of birth, but increases rapidly during childhood and adolescence. It may rise throughout life, provided an individual cultivates it. It may be damaged by neglect, alcohol or dugs, etc. It may also be hurt by social surroundings that are not supportive enough, over demanding, or frankly destructive. Therefore social support for health is crucial [6].

The demands of life are those that we have to respond to in order to lead a healthy life. (They must not be confused with demands a person may have toward life. These have nothing to do with the Meikirch Model.) A baby and a child are cared for by their social surroundings, such as mother, father, siblings, teachers, etc. During their productive years all individuals have to contribute to the society. Pensioned elderly persons are again supported by the society. Consequently, the demands of life vary continuously with the age of a person. At the same time the culture of a society modifies these demands, a factor which needs to be taken into consideration. A final important aspect is personal responsibility of each individual for his own health.

In each person the decision about health or disease depends on the balance between the total potential and the demands of life (Figure 2). If the two partial potentials combined outweigh the demands of life, a person is healthy. In contrast, if they weigh less, the person is diseased.

Within this context it is important to appreciate the nature of these potentials and why they are essential. At the time of birth the biologically given potential is the result of the genetic equipment and of the quality of the pregnancy. It consists of all biological aspects of life, which need to be protected, properly cared for and treated responsibly throughout life. The personally acquired potential is more difficult to understand. It comprises not only every capability a person has learned, but also some physical aspects that are acquired while learning. The capability of forming immune reactions, e.g., is biologically given, but the immunities we have are acquired. Obviously, emotional maturity and spiritual growth are part of the personally acquired potential. Finally, the way we overcome misadventures, injuries, and possibly severe harm may support or damage our personally acquired potential.

A good example to illustrate the contribution of each potential to the health of an individual is paraplegia resulting from an accident to the spinal cord. Such a condition (until now) represents an irreversible damage to

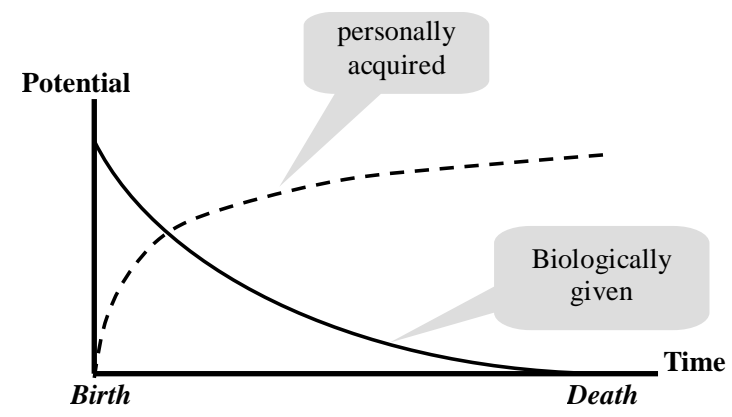

Figure 1. The potential of a person consists of two components, a biologically given and a personally acquired potential. In the graph the two lines are drawn arbitrarily, yet they show that during a lifetime the contribution of each component to the total potential varies continuously.

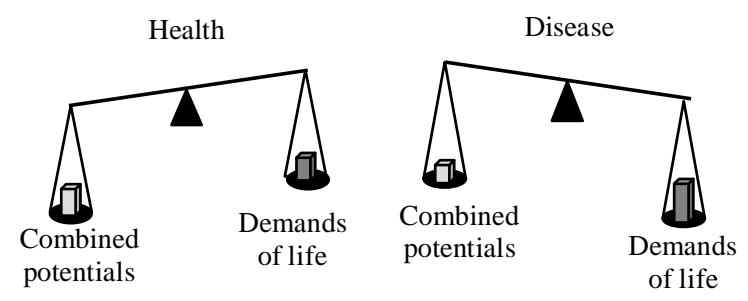

Figure 2. The decision about health or disease depends on the balance between the sum of the combined potentials and the demands of life. As with the decision about day and night the distinction between health and disease is clear in a majority of cases, but in some instances it may be blurred. 
the biologically given potential. Yet, through rehabilitation, which is exclusively concerned with the personally acquired potential, such persons may again become independent and even professionally active. It shows that the personally acquired potential may in part compensate for reductions in biologically given potential. This fact creates hope and supports patients with chronic diseases because they realize that they can do something about their condition, even when improvement of their physical state can no longer be expected. In a similar way the personally acquired potential gives hope to the elderly.

In order to fully appreciate the Meikirch Model, some further details need to be understood:

1) A relatively large number of healthy persons complain about discomfort and pains [7]. Consequently, health does not imply a "state of complete physical, mental and social well-being", because most healthy persons regularly have some complaints. Yet, they know how to deal with them. This observation further supports the idea that health is related to an ability to master the demands of life.

2) Chronically ill persons or humans with e.g. a metabolic or an immunological shortcoming may need long term treatment in order to lead a "healthy" life. For this reason two states of health must be distinguished, one without treatment and the other with a long term therapy, such as in hypothyroidism, diabetes mellitus, arterial hypertension, rheumatoid arthritis, etc.

3) The term potential may be somewhat elusive to grasp because it has to do with the future of a person. It must, however, be realized that medicine and health care are not so much concerned with the present but with an improvement of each person's future. The very purpose of medicine and public health is to preserve or restore whenever possible the immediate, medium, and long term future of patients and citizens. For these reasons the concept of "potential" is more appropriate than e.g. "condition".

\section{THE IMPORTANCE OF A DEFINITION OF HEALTH FOR MEDICINE AND HEALTH CARE}

"In any field, improving performance and accountability depends on having a shared goal that unites the interests of all stakeholders. In health care, however, stakeholders have myriad, often conflicting goals including access to services, profitability, high quality, cost containment, safety, convenience, patient-centeredness, and satisfaction. Lack of clarity about goals has led to divergent approaches, gaming of the system and slow progress in performance improvement.” With this introduction Michael Porter pleads for his concept of values, which he defines as health outcomes achieved per dollar spent (Figure 3) [8]. The problem of divergent objectives is well known in business organizations. In fact, experienced leaders take great care to identify and explain the purpose of their institution. By communicating it they intend to reach a higher level of cooperation and performance. In health care the overriding goal is obvious. All persons must work for the health of the people. Therefore it is now of critical importance to urgently achieve a consensus about what health is and to communicate it. It is our understanding that at present the Meikirch Model is the best available response to this need.

Another independent method to appreciate the importance of a definition of health may be derived from the social systems theory [9]. Table 1 shows some essential

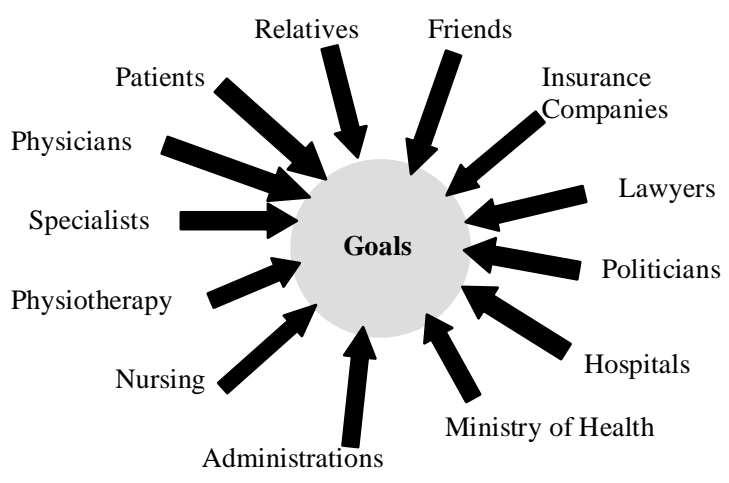

Figure 3. Lack of a common goal divides stakeholders in the health care system: As many goals are pursued as there are persons involved. Presumably this is a central reason for the different crises and the slow progress of health care systems.

Table 1. Comparison of some essential features of medicine and health care with selected examples of other social systems. Each system responds to demands by specific actions. Whether or not an action takes place is governed by the generalized symbolic medium of communication. In the case of medicine and health care this medium has no specific meaning, because health and disease have, up till now, remained undefined. Therefore, actions within this system occur much more arbitrarily than in the other social systems. This is damaging.

\begin{tabular}{|c|c|c|c|}
\hline System & Demands & $\begin{array}{c}\text { Generalized } \\
\text { symbolic medium } \\
\text { of communication }\end{array}$ & $\begin{array}{l}\text { Specific } \\
\text { Actions }\end{array}$ \\
\hline Economy & $\begin{array}{l}\text { Goods, } \\
\text { Services }\end{array}$ & $\begin{array}{c}\text { Money } \\
(\$, £, €, \text { CHF })\end{array}$ & $\begin{array}{l}\text { Purchase or } \\
\text { no Purchase }\end{array}$ \\
\hline $\begin{array}{l}\text { Legal } \\
\text { System }\end{array}$ & Justice & $\begin{array}{l}\text { System } \\
\text { of laws }\end{array}$ & $\begin{array}{l}\text { Agreement, } \\
\text { Court Order }\end{array}$ \\
\hline Science & $\begin{array}{c}\text { New } \\
\text { Knowledge }\end{array}$ & $\begin{array}{c}\text { Peer } \\
\text { Review }\end{array}$ & $\begin{array}{l}\text { Financing, } \\
\text { Publication }\end{array}$ \\
\hline $\begin{array}{l}\text { Medicine, } \\
\text { Health Care }\end{array}$ & $\begin{array}{l}\text { Improved } \\
\text { Health }\end{array}$ & $\begin{array}{l}\text { Health and Disease } \\
\text { (undefined so far) }\end{array}$ & $\begin{array}{l}\text { Diagnosis, } \\
\text { Treatment }\end{array}$ \\
\hline
\end{tabular}


features of a system. Each of them responds to demands that induce actions. Whether or not this action occurs is governed in each specific case by a generalized symbolic medium of communication. For instance, in an economy, people demand goods and services. Whether these are purchased or not depends on money. Only if the price is adequate for the goods or services, are they bought. In the legal system there may be a demand for justice, which can be responded to by an agreement or by a court order. Both cases must conform to the law in order to be valid. Therefore, in the legal system, the laws play the role of the generalized symbolic medium of communication. In science there is a demand for new knowledge. For both, the financing of projects and for their publication in scientific journals peer review is required. These examples may illustrate two features of a generalized symbolic medium of communication: It plays a central role for the processes that occur within the system and its nature is specific for each system. In medicine and health care by necessity the concept of health and disease serves as symbolic medium of communication. Consequently, it is pivotal for the health care system not to leave health and disease within the realm of arbitrary individual judgments, but to define them in such a way that everyone can agree about them. If in health care money would serve as generalized symbolic medium of communication, medicine would become part of the economy and lose its specific nature.

\section{WHY CHOOSE THE MEIKIRCH MODEL?}

When opting for a definition of health, it is important to compare it with the best possible alternatives. For lack of space only two eminent examples are discussed here:

1) Christopher Boorse [10] proposed a biostatistical value-free definition. He explains "health as the absence of disease" and "disease as a type of internal state, which is either an impairment of normal functional ability, i.e. a reduction of one or more functional abilities below typical efficiency, or a limitation of functional ability caused by environmental agents". Normal functional ability is defined by statistical comparison with an age and sex specific reference group. The value of this definition of health and disease consists in the fact that it is based on measurements and statistics. It has been criticized, however, on the basis that the selection of a reference group is not truly value-free, but requires a normative judgment. In addition the definition does not structure the health problem of patients. If a statistical significance of $\mathrm{p}<0.05$ is chosen, $5 \%$ of normal subjects are diseased. In addition, to define health, it is needed to measure the appropriate parameter. In practice this is difficult.

2) Lennart Nordenfelt [11] proposes a welfare theory of health. He considers health to be the primary concept in the web of medical thinking. He states: " $A$ is completely healthy if, and only if, $\mathrm{A}$ is in a bodily and mental state which is such that A has an ability to realize all his or her vital goals, given accepted circumstances.” This ingenious definition is normative and derived from the action theory. For the purpose of medicine and health care, though, the terms he uses are difficult to apply in practice. How do we assess e.g. vital goals or accepted circumstances?

3) Several other normative definitions use among other criteria the need or lack of need for medical care as a decisive factor to define health or disease. This leads to circular reasoning because disease in turn justifies by itself the administration of medical care.

The Meikirch Model is also normative. Its practical importance lies in the fact that all six criteria for the description of health or disease may be assessed in consultations with patients. Therefore they may serve as criteria to analyze value in health care, i.e. health outcome per dollar spent [12]. In addition, it structures the components of health in such a way, that they may be used in research, in advising individual patients, in medico legal expertises and in policy decisions for public health. The Meikirch Model may also serve to explain the nature of medicine to members of other social systems, e.g. economists, managers, politicians, lawyers, and scientists. If they understand the model, it may provide the common denominator for joint policy decisions [13]. This is particularly pertinent, because health care continuously evolves together with the other social systems. If, as postulated by Porter [12], the measurement of value must be its fundamental goal and improving value the driving force for every participant, the Meikirch Model could become a pivotal reference point for this purpose.

As a next step the different stakeholders need to be able to perceive the usefulness of the Meikirch Model and to agree to apply it for interdisciplinary communication about health care. For this purpose it probably is useful to start with a widespread discussion on this subject. More details may be found on a website [5]. Once the Meikirch Model corresponds to a general understanding, it is expected that medicine and health care will tangibly be more beneficial socially.

\section{CONFLICT OF INTEREST STATEMENT}

There are no funds involved from any side. For both authors there are no conflicts of interest.

\section{REFERENCES}

[1] Grad, F.P. (2002) Preamble of the constitution of the 
World Health Organization. Bulletin of the World Health Organization, 80

doi: 10.1590/S0042-96862002001200014

[2] Franke, A. (2006) Modelle von Gesundheit und Krankheit. Hans Huber Hogrefe AG, Bern.

[3] Bircher, J. and Wehkamp, K.-H. (2006) Das ungenutzte Potential der Medizin. Rueffer and Rub Publ, Zürich, 44-77.

[4] Bircher, J. (2005) Towards a dynamic definition of health and disease. Medicine, Healthcare \& Philosophy, 8, 335341. doi:10.1007/s11019-005-0538-y

[5] Bircher J. and Wehkamp K.H. (2011) Gesundheit und Medizin. 10 Thesen-Wie kann ein Gesundheitswesen funktionieren, wenn niemand sagen kann, was Gesundheit ist? Monsenstein und Vannerdat Verlag, Münster, Germany.

[6] Marmot M. and Wilkinson, R.G. (1999) Social determineants of health. Oxford University Press, Oxford.

[7] Lieberherr, R., Marquis, J.F., Storni, M. and Wiedenmayer, G. (2007) Gesundheit und Gesundheitsverhalten in der Schweiz 2007. Schweizerische Gesundheitsbe- fragung, Neuchâtel, Switzerland, Bundesamt für Statistik.

[8] Porter, M.E. (2010) What is value in health care? The New England Journal of Medicine, 363, 2477-2481. doi:10.1056/NEJMp1011024

[9] Chenilo, D. (2002) The theorization of social co-ordinations in differentiated societies: The theory of generalized symbolic media in Parsons, Luhmann and Habermas. British Journal of Sociology, 53, 431-49. doi:10.1080/0007131022000000581

[10] Boorse, C.A. (1997) Rebuttal on health. In: Humber J.M. and Almeder R.F. Ed., What is disease, Humana Press, Totowa NJ, 1-134.

[11] Nordenfelt, L. (2001) Health, science and ordinary language. Editions Rodopi BV, Rodopi, New York.

[12] Porter, M.E. (2008) Defining and introducing value in health care. In: McClellan M.B. Ed., Evidence-Based Medicine and the Changing Nature of Healthcare: Meeting Summary, National Academies Press, IOM, 161-72.

[13] Savigny, de D. and Adam, T. Ed. (2010) Systems thinking for health care strengthening. Advances in bioinformatics, 2, p. 268925. 